\title{
Stabilization of the vertical take-off of a rigid flying wing for an airborne wind energy system
}

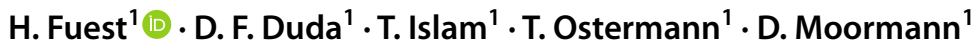

Received: 8 January 2021 / Revised: 22 July 2021 / Accepted: 17 August 2021 / Published online: 13 September 2021

(c) The Author(s) 2021

\begin{abstract}
Airborne wind energy is an emerging technology that harvests wind energy with flight systems connected via a tether to the ground. In the project "EnerGlider", a flying wing is meant to fly fully automated by its own control units. This contribution discusses the challenges to control and trim this flying wing during vertical take-off and landing under the influence of a horizontal wind velocity. High wind velocities can lead to unstable and untrimmed states concerning the longitudinal motion of the flying wing. The paper analyzes the influence of design modifications of thrust vector and elevon area to enhance the flight envelope of the trimmed states to higher wind velocities. Besides, the tether force as additional control unit is considered for strong wind forces. It is demonstrated that a tether force acting behind the center of gravity can significantly enhance the robustness of the flight system concerning wind velocity. Moreover, the unstable flight states emerging during vertical take-off and landing can be stabilized with a flight control.
\end{abstract}

Keywords Airborne wind energy $\cdot$ Vertical take-off and landing $\cdot$ Flying wing $\cdot$ Trim states

\section{List of symbols}

AWE Airborne wind energy

VTOL Vertical take-off and landing

A System matrix of state-space matrix

$B \quad$ Input matrix of state-space matrix

$F_{T} \quad$ Tether force

$K \quad$ Controller gain

$k \quad$ Factor within PD-controller

$l_{\kappa} \quad$ Elevon's depth

$l_{\mu} \quad$ Reference chord length

$M_{\text {pitch }} \quad$ Pitching moment

$q \quad$ Pitch rate

$S_{\text {disk }} \quad$ Propeller area

$u \quad$ Body velocity in $x$-direction

$\vec{u} \quad$ Input vector of the flight system

$u_{A} \quad$ Inflow velocity

$V_{I I I} \quad$ Inflow velocity of element III

$w \quad$ Body velocity in $z$-direction

$x, y, z \quad$ Components of position vector

$\vec{x} \quad$ State vector of the flight system

$X, Z \quad$ Forces in $x / z$-direction

H. Fuest

fuest@fsd.rwth-aachen.de

1 Chair and Institute of Flight System Dynamics, RWTH Aachen University, Aachen, Germany
$x_{T} \quad$ Tether point of attack

$z_{\text {off }} \quad$ Lever arm for motors

$\theta \quad$ Pitch angle

$\kappa \quad$ Elevon deflection

$\rho \quad$ Density of air

$\sigma_{T} \quad$ Tilt angle of thrust vector around $y_{b}$-axis

()$_{b} \quad$ Body coordinates

() Commanded

()$_{g} \quad$ Geodetic coordinates

\section{Introduction}

Airborne Wind Energy (AWE) is an emerging technology aiming at harvesting wind energy with flight systems tethered to the ground. This technology holds great potential for increased energy output, as the system only consists of a generator station on the ground, at least one tether and an airborne flight system, which operates at high altitudes with strong and constant wind conditions.

The Institute of Flight System Dynamics conducts research in this field in the research project "EnerGlider". Within this project, a flying wing that is supposed to fly fully automated by means of its own control elements is used as airborne vehicle. For this type of Airborne Wind Energy System, the aerodynamic lift force at the wings of 
the airborne vehicle is transmitted mechanically via the tether to the ground, where an unwinding of the tether from a winch drives a generator and generates electrical energy. Such a configuration makes use of the high aerodynamic performance during flight, while also enabling vertical take-off and landing (VTOL). During energy production in tethered crosswind flights, the flight system operates as a glider, where the forward component of the aerodynamic force is used to accelerate. Hence, propellers are not necessarily required for this flight phase. Still, the aircraft has to perform the VTOL and a transition into the crosswind flights and back, for which a propulsion system is vital.

This take-off and landing procedure is a crucial flight phase within Airborne Wind Energy projects. Currently, for the take-off and landing procedure of the airborne vehicle, different solutions such as catapult launching like systems on a rail [1] or with a cantilever [2,3] exist. However, these configurations exclude the possibility to operate the flight system independently from the ground station. To control the flight system independently during VTOL, a propulsion system is considered in the design. Thus, this flight system is able to take-off and land vertically as a tailsitter, as well as transitions into forward flight and back by use of its own propulsion system.

The elevons are the only control units to control roll and pitch moment. Therefore, many flying wings are constructed with a large proportion of the elevons area in relation to the wing area to control the aircraft [4-6]. This increases their effectivity, since an elevon deflection results in a greater change of the local lift force at the elevons. However, as the body velocity is small in hover mode during vertical takeoff, the inflow of the elevons is resulting mainly from the propeller slipstream. Therefore, only the part of the elevon in the slipstream is relevant for lift generation. A further enlargement of the elevons broader than the area behind the slipstream of the propeller does not increase the elevons effectivity. As the flying wing has no horizontal tailplane for longitudinal stability, the elevons are the only control unit to counteract the pitching moment induced by the aerodynamic inflow. This is especially challenging for operations with high wind velocities, for which aircraft of AWE-systems have to be designed for. The superposition of small upward velocities during VTOL with high horizontal wind velocities acting vertically on the flying wing can introduce high pitching moments leading to an unstable and untrimmed flight state. Hence, the longitudinal stabilization of the vertical take-off of a flying wing under influence of wind is of further importance and investigated within this paper. This paper tries to answer the research question on how to increase the area of stable trimmed states of the longitudinal motion of a flying wing.

To analyze the flight behavior, Sect. 2 deals at first with the typical components and models of AWE-Systems with focus on the flight system. Its aerodynamic model is introduced based on an existing semi-analytical approach [7]. In Sect. 3, this model is discussed by analyzing the stability of the trim states of the longitudinal motion of the flying wing. Moreover, design options to expand the flight envelope for trimmed states are introduced. As the flight system is longitudinally unstable during vertical take-off, a simplified closed-loop control concept of the flight system is presented for stabilization.

\section{Components and models of the AWE-system}

The AWE-system can be decomposed into the three main components flight system, tether and ground station with a generator unit (Fig. 1).

The flight system is operated in a crosswind flight, where the induced aerodynamic forces are transmitted via the tether to the ground station. As the flying vehicle operates in this crosswind flight and gains distance to the ground station, the tether is unwound from a tether drum inducing a rotation of a joined generator shaft. After the maximum tether length is reached, the flight system is flying in its initial position and the process of energy generation restarts.

Flight system and tether are modeled to simulate the forces and the flight behavior of the system. The model of the tether to calculate the tether forces is based on a hybrid approach consisting of a rod model with tensile forces and a catenary model considering an external line load [8]. This external load takes into account the earth's gravitational field and aerodynamic loads induced by the body velocity of the tether and the wind.

The focus of this paper is on the flight system. Its characteristics is influenced by the tether force acting at the flight system. To achieve a high power output of the AWE-System, the flight system is intended to have a high lift-to-drag ratio. As a flying wing has such high lift-to-drag ratio, while also

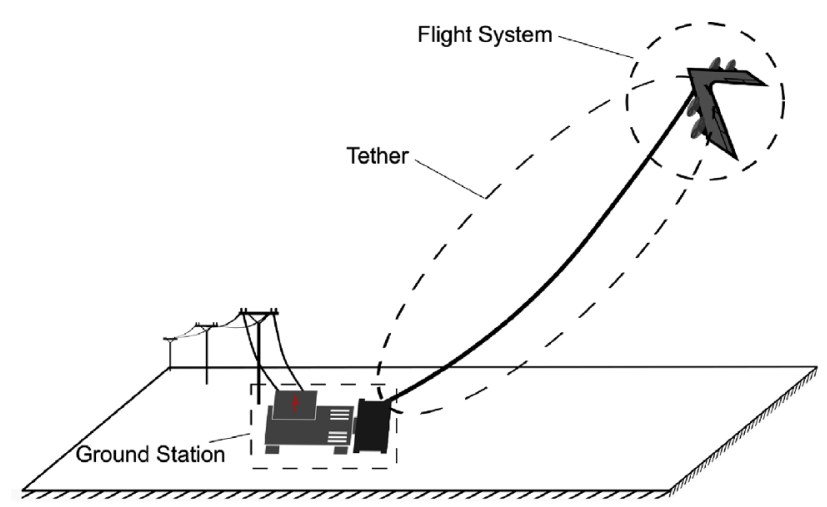

Fig. 1 AWE-components 
offering the possibility to take-off vertically, it is chosen as configuration for this flight system. The initial demonstrator of this system has a wingspan of $4.4 \mathrm{~m}$ and a mass of $13 \mathrm{~kg}$.

The control of the flying wing is ensured by four separate elevons and four propulsion units (Fig. 2). The propulsion units provide thrust during the VTOL phase of up to $180 \mathrm{~N}$. The elevons are located downstream of the propeller units to increase their efficiency during VTOL. In the current configuration, the steering surfaces can be deflected up to $30^{\circ}$ in each direction. When the system reaches the tethered crosswind flight, the acceleration is obtained by the forward component of the lift force due to the wind velocity. At this point, the propellers are supposed to fold in to reduce drag.

Figure 3 depicts the control method of the flying wing. During energy generation, the flight system is controlled in all axes only with its elevons. A uniform deflection of all elevons induces a pitch moment, while an asymmetric deflection on both sides induces a roll moment. A yaw moment can be induced by opposed deflection of the elevons on one side to increase drag one-sided. During VTOL however, the yaw moment can be induced more effectively by differential thrust of the propulsion units.

In the following, the body-fixed and geodetic coordinate systems are utilized to describe the motion of the flight system (Fig. 4). The geodetic $z_{g}$-coordinate points into the

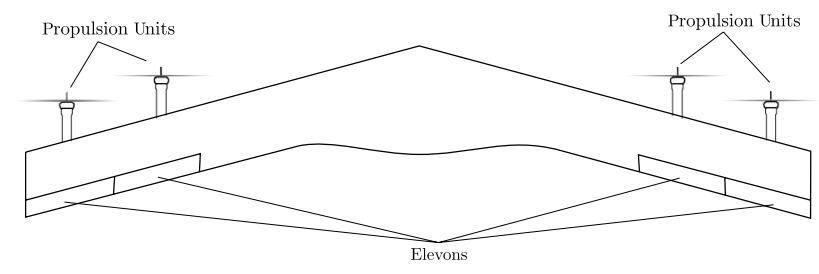

Fig. 2 Sketch of the EnerGlider control units

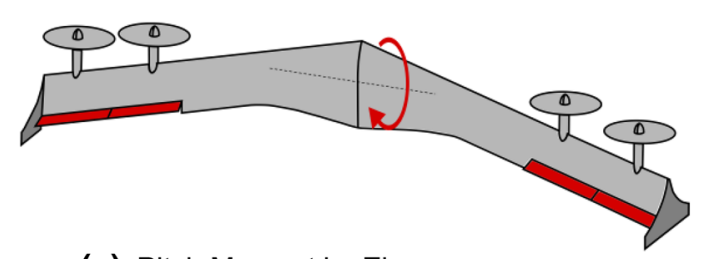

(a) Pitch Moment by Elevons

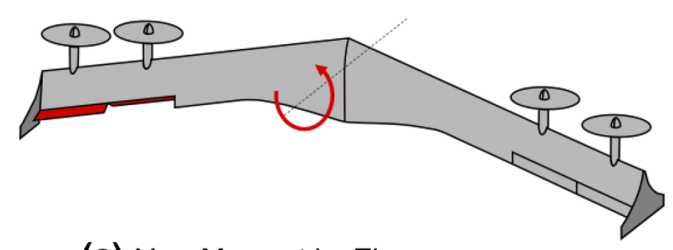

(c) Yaw Moment by Elevons

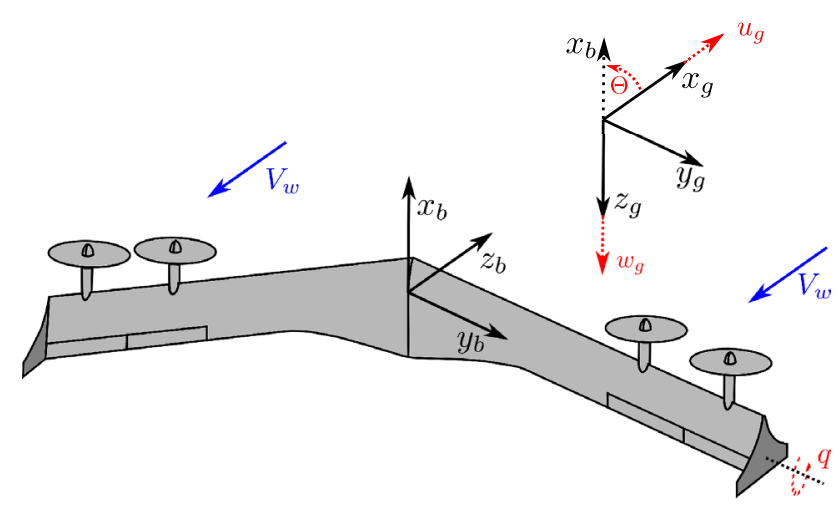

Fig. 4 Body-fixed and geodetic coordinate systems

earth's center and the $x_{g}$-coordinate is aligned northward. In the body-fixed system, $x_{b}$ is defined in direction of the flight system's nose, the $y_{b}$ axis is in span direction and the $z_{b}$ axis rectangular to $x_{b}$ and $y_{b}$. The flight system's states of the longitudinal motion (body velocities $u_{g}$ and $w_{g}$ in geodetic $x_{g}$ and $z_{g}$ direction, as well as pitch rate $q$ and pitch angle $\Theta$ ) are depicted in red, dashed lines. The flight system is assumed to be aligned with its $z_{b}$-coordinate into the wind field with wind velocity $V_{w}$.

The aerodynamics of the flying wing are modeled in a semi-analytical element based approach, which is developed for tilt-wing aircraft with angle of attacks from $-180^{\circ}$ to $180^{\circ}[7,9]$. The approach separates the wing into elements with similar aerodynamic characteristics. The flying wing is separated into elements consisting of a clean wing area with a free stream (I), a part with aerodynamic influence of the elevons (II) and the elevon and wing elements that are within the slipstream of the propeller (III) (Fig. 5).

The aerodynamic loads acting on the clean wing elements are calculated using the polar of the airfoil, extrapolated to

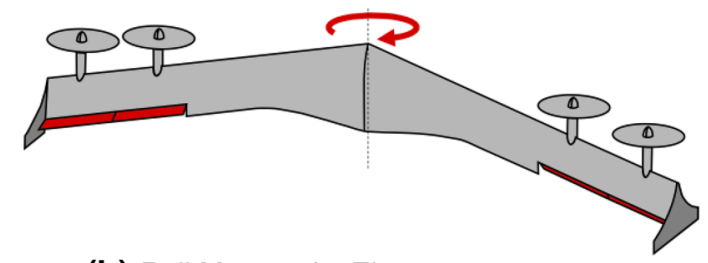

(b) Roll Moment by Elevons

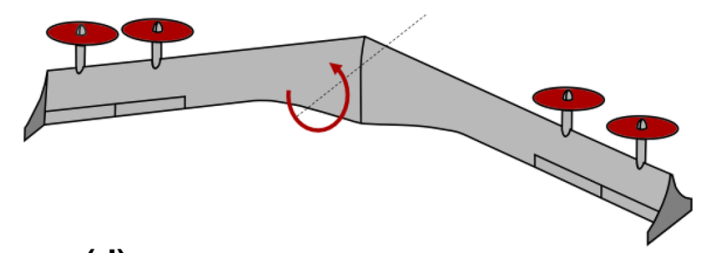

(d) Yaw Moment by Thrust

Fig. 3 Control elements of the flight system. a Pitch moment by elevons. b Roll moment by elevons. c Yaw moment by elevons. d Yaw moment by thrust 


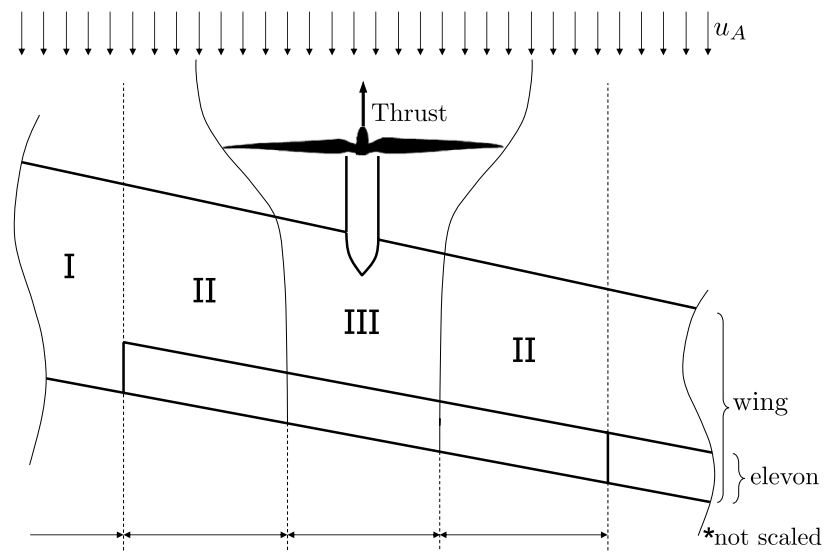

Fig. 5 Wing elements of the flying wing, not scaled [11]
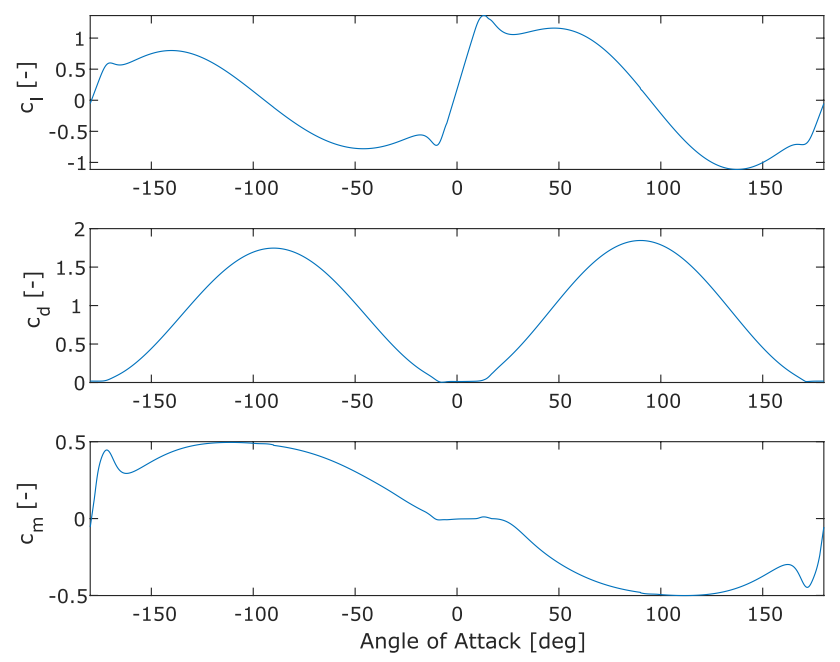

Fig. 6 Polar of the airfoil

$180^{\circ}$ by use of the Montgomerie approach [10]. In Fig. 6, lift, drag and pitch moment coefficients are presented for the flying wing. The lift coefficient reaches its maximum of 1.4 at an angle of attack around $14^{\circ}$ and decreases for higher angles. However, as drag increases up to a vertical inflow of $90^{\circ}$, the pitch moment increases as well.

Due to the slipstream, the inflow speed at the wing located behind the propeller is increased, following Eq. (1), resulting from the Bernoulli-equations along a streamline [11]:

$V_{I I I}=\sqrt{\frac{T_{\text {Motor }}}{S_{\text {Disk }}} \cdot \frac{2}{\rho}+u_{A}^{2}}$.

Here, $T_{\text {Motor }}$ corresponds to the effective thrust of the propeller with propeller area $S_{\text {Disk }}$, reduced by the influence of the inflow velocity $u_{A}$. To reduce complexity in this first design stage, only the axial inflow velocity is regarded to calculate the slipstream velocity. The density of the airflow is respected by $\rho$. As this equation corresponds to a completely developed wake, a contraction factor is applied to calculate width and induced velocity at the aerodynamic center of the wing [12].

The aerodynamic coefficients of the wing are modified in the case of an elevon deflection. Thereby, the elevon is modeled as plain flap. Depending on deflection angle, angle of attack and flap to chord ratio, empirical correction factors are applied [9].

Considering the aerodynamic profile of the airfoil and the modifications due to elevon and propeller, the aerodynamic loads can be computed for all elements representing the flight system. The resulting aerodynamic loads are reduced to the center of mass of the entire flight system and considered within the equation of motion to derive the nonlinear model of the flight system's motion.

In hover mode, during take-off or landing, the body velocity is low, leading to a negligible inflow velocity. Only the wing parts exposed to the slipstream of a propeller, considered by element (III) shown in Fig. 5, experience an aerodynamic load that can be controlled by a deflection of the elevon. However, even for these elevon elements, the inflow velocity remains low, leading to a decrease of the elevon effectivity during the VTOL phase. Still, wind velocities acting with angle of attacks up to $90^{\circ}$ can induce strong pitch moments at the flight system (compare with Fig. 6). Hence, issues to trim the flight system are expected. At these high angle of attacks, inaccuracies of the model can occur, as vortexes and flow separation require advanced analyses like computational fluid dynamic (CFD) simulations. Yet, this approach allows to receive tendencies for trim states and the system's stability and enables to analyze the influence of design modifications.

\section{Results}

The model of the flight system is analyzed with respect to its trim states and its stability of the longitudinal motion. To increase the area of trimmed states, system modifications and their influence and applicability are discussed. The dynamic stability of the system is investigated by analyzing the poles. As the trim states during VTOL are unstable, a simplified closed loop system is introduced to stabilize the poles.

\subsection{Discussion of the flight system model}

As it is discussed in Sect. 2, the effectivity of the elevons during take-off is reduced. This is critical mainly concerning the pitch moment, as the elevons have to compensate the pitch moment induced by the aerodynamic forces on 
the entire wing. Regarding the yaw moment, it is induced by differential thrust which is not affected by the low inflow velocity and the roll moment modifies the orientation of the flight system and is hence not decisive for its stability. Thus, the focus is on the pitch moment and the longitudinal motion in the following. To evaluate the effectivity of the elevons, the stationary, longitudinal trim states of the flight system are calculated. A longitudinal trim state exists when all forces in $x$ - and $z$-direction, as well as the pitch moment $M_{\text {Pitch }}$ are equalized for a static state, see Eq. (2):

$$
\sum X=0 ; \quad \sum Z=0 ; \quad \sum M_{P i t c h}=0 .
$$

The flying wing's longitudinal motion is defined explicitly by the four state variables pitch rate $q$, body velocity in geodetic X-direction $u_{g}$, velocity in z-direction $w_{g}$ and pitch angle $\Theta$. The velocity in geodetic coordinates is applied to have a better interpretability than in body coordinates. For a stationary trim state, the state variable $q$ can be considered as zero. Yet, to analyze stability of each trim point, the nonlinear model of Sect. 2 is linearized around the corresponding trim point, with respect to the pitch rate $q$ as well.

The flight system is equipped with two control units for longitudinal motion, thrust to control the velocity and elevons to control the pitch moment. The flight system is fully controllable around all axes with these control units.
Two of the three steady flight states can be prescribed while the third one arises from the trim condition.

In the following, these trim states of the longitudinal motion are analyzed for a defined interval of velocities $u_{g}$ and $w_{g}$ in geodetic coordinates. For each combination of $u_{g}$ and $w_{g}$, the forces and moments as nonlinear function of the deflections of the elevon and the throttle position are minimized for a possible trim condition, whereby a corresponding pitch angle is obtained. The velocity $u_{g}$ can hereby be interpreted as body velocity in geodetic $x$-direction or as wind velocity in negative $x$-direction for a fixed position of the flight system. By linearizing the plant for each operating point, the stability of the flight system in the corresponding trim conditions can be analyzed and evaluated.

The result is depicted in Fig. 7. For illustration purposes, the ordinate of the diagram is reversed, as a negative value of $w_{g}$ denotes an upward movement. It is assumed that the flight system is oriented into the wind field to avoid a negative inflow.

All red markers (plus ' + ' and circle 'o') describe a state, where the trim condition cannot be met, while the green and yellow stars (' 2 ' ' and ' $*$ ') define a trimmed condition. Untrimmed conditions occur at high flight velocities, since thrust of the propellers is not sufficient, compare with area 1 in Fig. 7. Also for high downward velocities at low horizontal velocities $u_{g}$, as thrust would need to be negative to meet the trim condition (compare with red circles). Besides, the corresponding inflow velocities for insufficient elevon deflection can be identified (area

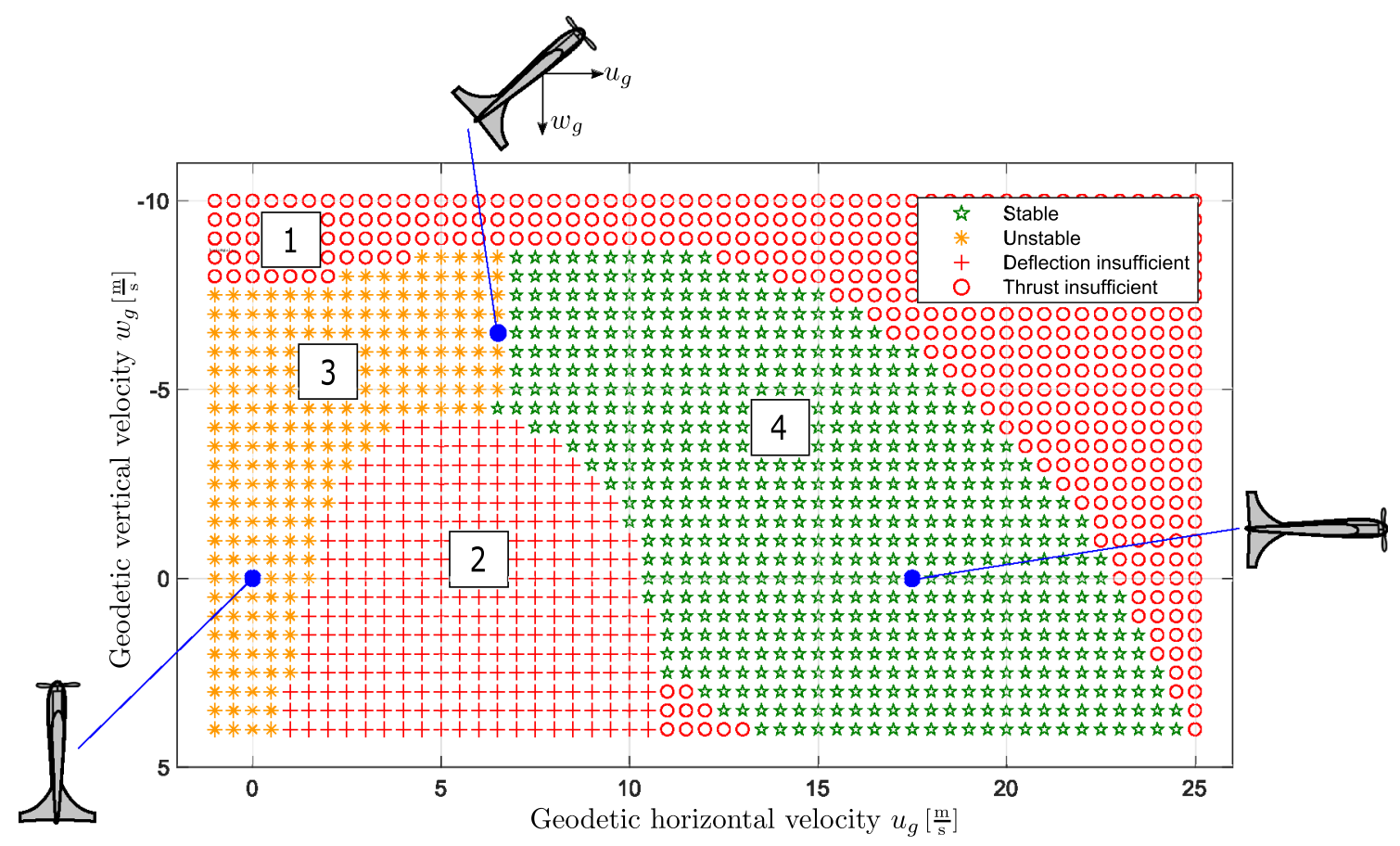

Fig. 7 Simulated, stationary trim states of the flying wing and their stability 


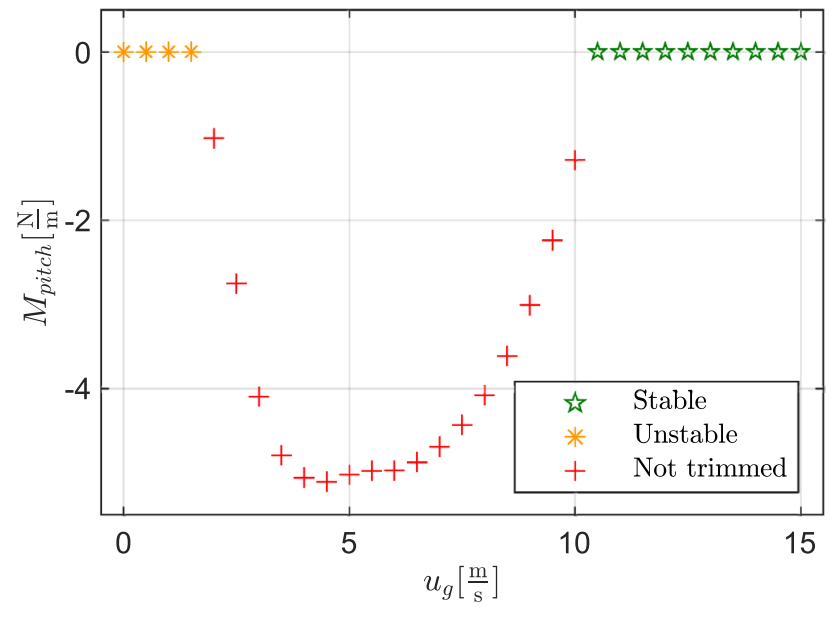

Fig. 8 Resulting pitch moment for $w_{g}=0 \mathrm{~m} / \mathrm{s}$

2). During vertical take-off, the flight system is oriented upwards with around $90^{\circ}$ nose up. A horizontal velocity leads to a vertical inflow in the body-fixed coordinate system. This can introduce a high pitch moment that cannot be counteracted by the elevons, as their effectivity is reduced at low inflow velocities. Figure 8 illustrates the residuum of the pitch moment $M_{\text {Pitch }}$ of the trim equations for variable horizontal velocities $u_{g}$ and at hover mode for $w_{g}=0 \mathrm{~m} / \mathrm{s}$. For all non-trimmed states, where the residuum is unequal zero, the remaining pitch moment for a maximum deflection of the elevons is illustrated. This resulting negative pitch moment due to a high angle of attack causes a nose-down motion that cannot be trimmed by the elevons. In a first design where an untethered VTOL is considered, these flight states at around $5-10 \mathrm{~m} / \mathrm{s}$ in hover mode with no vertical velocity do not necessarily require to be covered by the flight system. Still, a certain extent of robustness against the wind velocity is necessary for a safe flight performance. Hence, the extension of the flight envelope to higher velocities is part of the investigation within this contribution.

The trimmed states in Fig. 7 are classified as static unstable (area 3) and stable (area 4) flight states. For high horizontal velocities, the flight states are longitudinal static stable, meaning that small perturbations from the trimmed state induce a moment that acts in opposite direction to reduce these perturbations. Trim states exist for low horizontal velocities as well, however, there is no resetting moment to reduce deviations and hence it corresponds to an unstable, trimmed flight state.

The plant analysis of Fig. 7 indicates a low wind robustness, since it is not possible to trim the flight system for $2 \mathrm{~m} / \mathrm{s}$ wind velocity or higher in hover mode at $w_{g}=0 \mathrm{~m} / \mathrm{s}$. The pitch moments cannot be compensated by the elevons, as the rudder effectivity is limited. As a result, modifications have to be made to extend the flight envelope of the flight system.

With regard to applications of Airborne Wind Energy, it is possible to make use of the tether force as additional control unit. Besides, constructive adjustments of the flight system are analyzed. The elevons area can be enlarged to increase their effectivity or the thrust vector be adjusted. The influence of the mentioned modifications is analyzed in the following.

\subsection{Modifications to adjust the flight envelope}

To analyze the vertical take-off, the threshold between trimmed and untrimmed flight states is examined in detail. The trim states for these velocities are depicted in Fig. 9 as detailed view of Fig. 7 with focus on the threshold between untrimmed and trimmed states. For these velocities, Fig. 10 depicts the corresponding pitch angle of the trimmed state at $w_{g}=0 \mathrm{~m} / \mathrm{s}$. It can be identified that the pitch angle decreases for a higher horizontal velocity. The flight system adjusts its nose into the wind to equalize the wind forces. For increasing values, the elevons are not able to equalize the pitch moment for these high angle of attacks and the state cannot be trimmed.

In the following, the boundary between the states that can be trimmed and the area, where the elevon deflection is not sufficient, is investigated in detail. The objective is to shift the boundary to higher horizontal and lower vertical velocities to enhance the flight envelope of the flying wing. In doing so, constructive adjustments like an increase of the

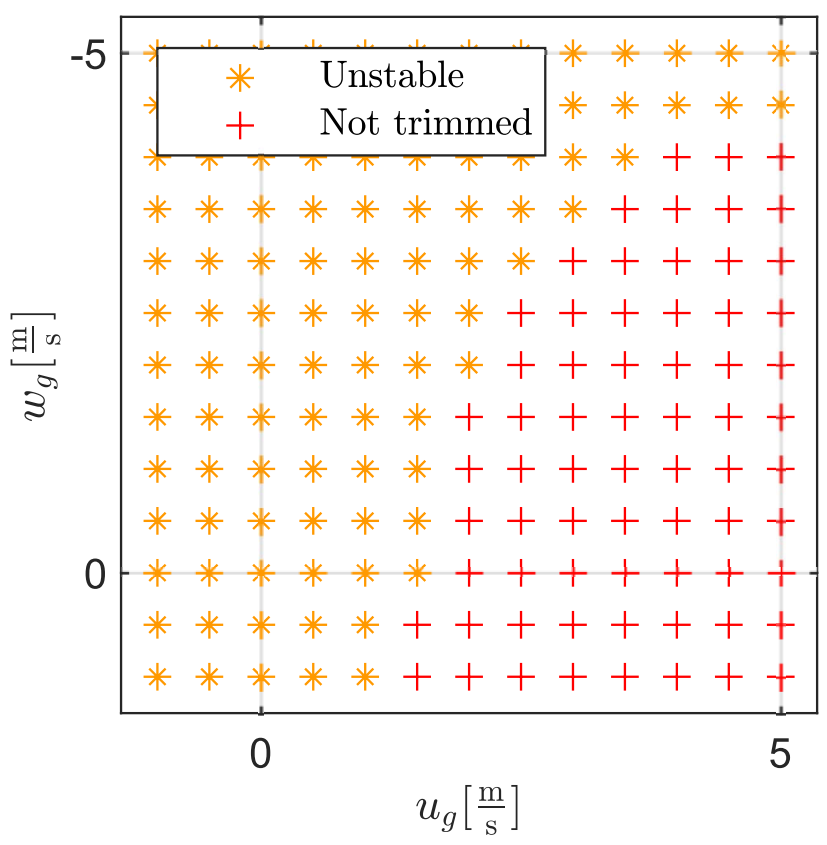

Fig. 9 Trim states during VTOL 


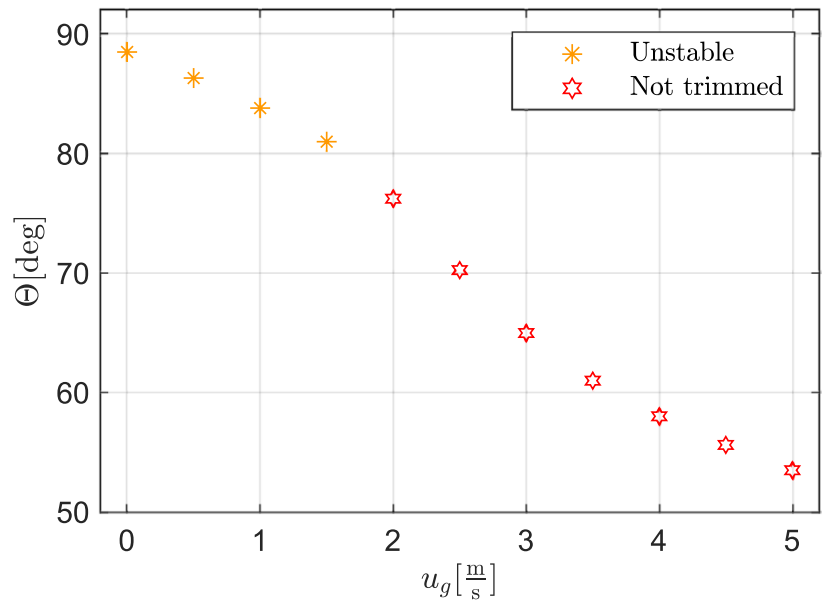

Fig. 10 Pitch angle at $w_{g}=0 \mathrm{~m} / \mathrm{s}$

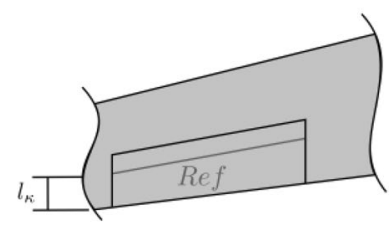

(a) Sketch

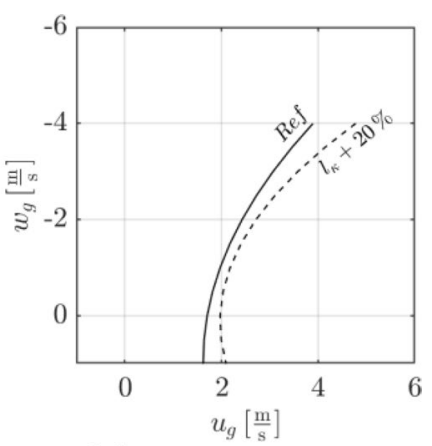

(b) Flight Envelope
Fig. 11 Modification of the elevon depth. a sketch. b flight envelope

elevon area and a modification of the thrust vector, as well as the influence of the tether force are analyzed.

Various adjustments of the flight system are investigated in order to analyze their influence on the trim states. Modifications to increase the flight systems ability to enhance the flight envelope are considered.

By enlarging the area of the elevons, the additional lift generated by a deflection and therefore the positive pitch moment is increased. This improves the effectivity of the elevons to induce higher control moments. As the elevon is placed in the center behind the slipstream of the propeller, only the depth of the rudder is enlarged. Figure 11a depicts the sketch of the modification of elevon length $l_{\kappa}$ and Fig. $11 \mathrm{~b}$ the relating result of the flight envelope for a modification of the rudder depth by $20 \%$ of the reference chord length $l_{\mu}$. The boundary line between trimmed and untrimmed states from Fig. 9 is portrayed depending on the velocities $u_{g}$ and $w_{g}$. To the right of this line, the flight system cannot be trimmed. It can be identified that for an increased rudder depth compared to the reference rudder

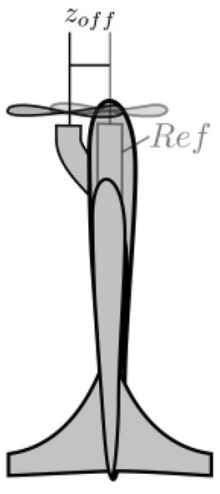

(a) Sketch

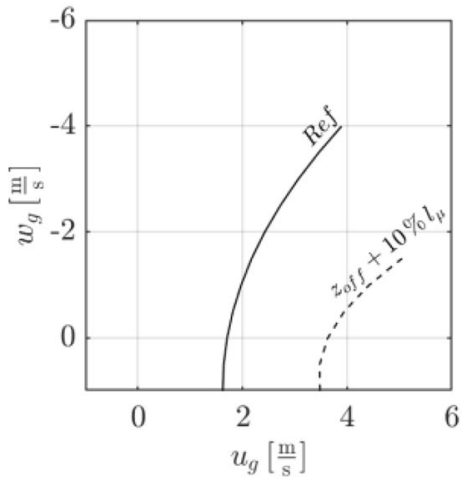

(b) Flight Envelope
Fig. 12 Modification of the thrust vector position. a sketch. b flight envelope

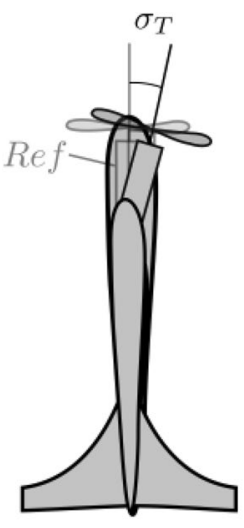

(a) Sketch

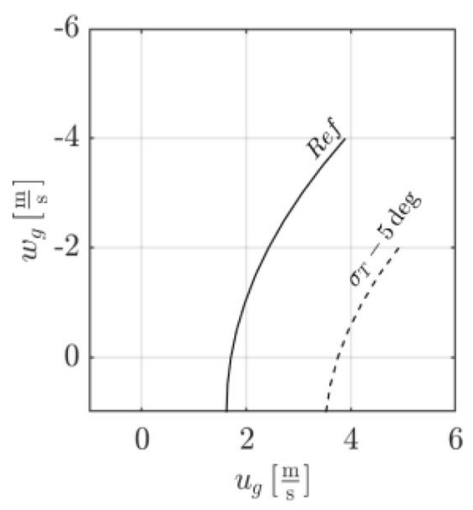

(b) Flight Envelope
Fig. 13 Modification of the thrust vector angle. a sketch. b flight envelope

area (Ref), the boundary shifts to higher horizontal velocities $u_{g}$. Nevertheless, its influence is compared to the high modification of the elevon of small extent, as the inflow velocity during VTOL is still low. The lift force generated by the elevons is proportional to the elevon area but quadratic proportional to the inflow velocity. Hence, the area enhancement cannot compensate for the low inflow velocity.

The thrust of the propellers, however, is not affected by the small inflow velocity. It is possible to induce a pitch moment by adjusting the thrust vector. Its angle of attack or position of attack in $z_{b}$-direction can be changed to induce a positive pitch moment. This is possible since the propellers are used only during VTOL and their modifications do not affect the energy generation phase.

Concerning a modification of the thrust vector, the results are pictured in Figs. 12 and 13. With a thrust point of attack by $10 \%$ of the reference length below the center of gravity $z_{\text {off }}$ (Fig. 12) or thrust tilted upwards with angle $\sigma_{T}$ (Fig. 13), 
a positive pitch moment is induced. The remaining negative pitch moment in the trimmed states (compare with Fig. 8) can be counteracted such that the flight envelope is enhanced considerably. However, an unlimited variation of the thrust vector below the center of gravity is not possible, as it is structurally challenging to place the propellers outside of the airfoil, whose thickness is defined by the aerodynamics.

So far, only the untethered flight states are considered. However, in the application as AWE-System, the tether force $F_{T}$ acting in the center of gravity can be utilized as further control input. At high wind velocities, the stretched tether can compensate a horizontal drift of the flight system. The flight system is able to pitch less into the wind direction, since the tether already accounts for keeping position. For a higher wind velocity, a stronger horizontal tether force is necessary. In the following, only this horizontal force is considered in a first approach. This corresponds to the takeoff position close to the ground. At higher flight altitudes, vertical forces have to be compensated by a higher thrust requirement of the flight system.

Figure 14 depicts the results for the reference case at $F_{T}=0 \mathrm{~N}$ and for horizontal tether forces of $80 \mathrm{~N}$ and $130 \mathrm{~N}$. Again, the trimmed area is on the left of the plotted lines. It can be identified that the flight envelope is enhanced for higher tether forces significantly. For a tether force of $80 \mathrm{~N}$, no untrimmed states exist below $w_{g}=-1.3 \mathrm{~m} / \mathrm{s}$ and at $130 \mathrm{~N}$, the threshold for insufficient elevons is shifted at $w_{g}=0 \mathrm{~m} / \mathrm{s}$ up to $u_{g}=5 \mathrm{~m} / \mathrm{s}$.

However, it has to be noted that the powertrain has to withstand the tether force. A high tether force also means a high thrust requirement, as the $z_{g}$-component of the tether force has to be equalized when the flight system is gaining height. This again results in a stronger lift force due to a higher slipstream velocity and hence a pitch moment that needs to be equalized by the elevons. For the maximum considered tether force of $130 \mathrm{~N}$, vertical tether forces exceed half of the hover thrust requirement at an elevation angle

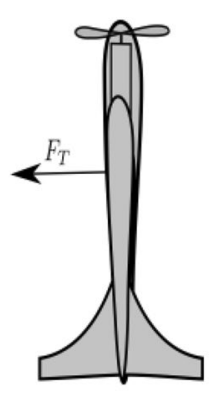

(a) Sketch

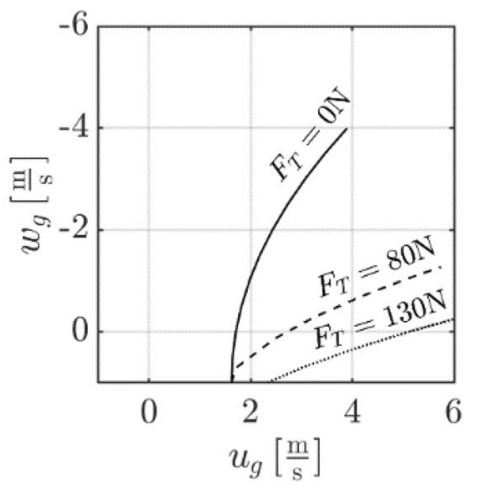

(b) Flight Envelope
Fig. 14 Influence of the Tether force. a sketch. b flight envelope of the flight system of $30^{\circ}$. At this point, the vertical tether forces mainly influence the thrust requirement and the trim states and can no more be neglected.

To overcome this issue, the point of attack can be shifted backwards to introduce a further pitch moment by the tether force. At high horizontal velocities, this equalizes the aerodynamic pitch moment. This allows to decrease the norm of the tether force for the same enhancement of the flight envelope. With a reduced tether force of $80 \mathrm{~N}$, the elevation angle of the position of the flight system rises to $45^{\circ}$, from where on the vertical forces have to be taken into account as well. This is expected to be the flight altitude, in which a transition into the energy generation phase will take place. Hence, the VTOL phase ends and the assumption of considering only the horizontal forces holds.

Figure 15 compares the case with a tether force of $80 \mathrm{~N}$ acting in the center of gravity as reference (Ref) and a backward offset of $x_{T}=-0.05 \mathrm{~m}$ backwards. It can be concluded that a small shift of the point of attack can further enhance the flight envelope. In hover mode at $w_{g}=0 \mathrm{~m} / \mathrm{s}$, the flight envelope is fully trimmed for this configuration for all horizontal velocities up to the maximum flight velocity. Only at downward vertical velocities, the flight system cannot be trimmed for horizontal velocities higher than $4 \mathrm{~m} / \mathrm{s}$.

It can be noted that alternative flight paths like rotating the flight system with span width in wind direction to reduce the induced pitch moment can be investigated as well. Besides, a corresponding trim tether force corresponding to certain wind velocities can be examined in a further analysis [13].

For all mentioned constructive modifications, the untrimmed area can be shifted to higher horizontal velocities. However, the elevon depth modification has relatively smaller effect compared to the other introduced adjustments. Best results can be identified for utilizing the tether force slightly behind the center of gravity to introduce a further

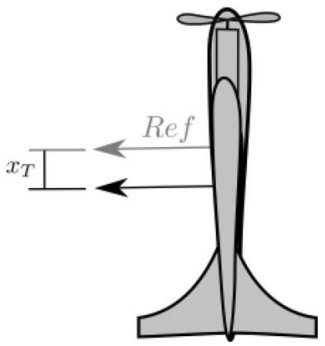

(a) Sketch

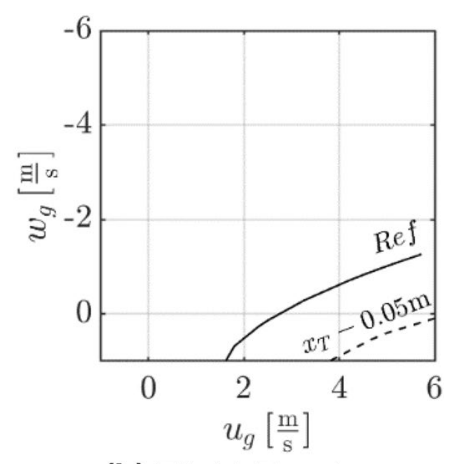

(b) Flight Envelope
Fig. 15 Influence of the Tether's point of attack. a sketch. b flight envelope 
pitch moment. In any case, the influence on the subsequent flight phases during crosswind flight has to be assessed.

\subsection{Longitudinal stability}

To analyze dynamic stability, the poles of the dynamic model are considered. The aerodynamic model of Sect. 2 is linearized around each trim state to receive a state-space system with states $\vec{x}$ of the longitudinal motion and input $\vec{u}$ of elevon deflection $\kappa$ and thrust $f$, see Eq. (3):

$\overrightarrow{\vec{x}}=A \cdot \vec{x}+B \cdot \vec{u} ; \vec{x}=\left(\begin{array}{c}q \\ \theta \\ u \\ w\end{array}\right) ; \vec{u}=\left(\begin{array}{c}f \\ \kappa\end{array}\right)$.

In Fig. 7, the trim points are classified as stable and unstable. As shown, the flight system is unstable during the VTOL phase at low velocities. The poles for the trim state at $w_{g}=u_{g}=0 \mathrm{~m} / \mathrm{s}$ are depicted exemplary in Fig. 16a. There exists a pole with a positive real component. The corresponding eigenvector has dominant parts in the states $q, w_{b}$ and $\Theta$. Having a perturbation in the body-fixed $z$-velocity $w_{b}$ , a pitch moment is induced and the flying wing tends to tilt around its $y$-axis (absolute value of $q$ and $\Theta$ increase). With the upward position of the flight system, the wind velocity is acting perpendicular with angles of attack up to $90^{\circ}$ on the flying wing and induces drag and the corresponding pitch moment that leads to the instability (compare with Fig. 6).

By comparing the poles with the longitudinal eigenmodes of conventional aircraft, the complex conjugated poles correspond to the short period mode, while the phugoid is split into the two first order lag elements. Here, the state of the flight velocity is still stable. However, the state flight angle corresponds to the unstable pole, which can be explained

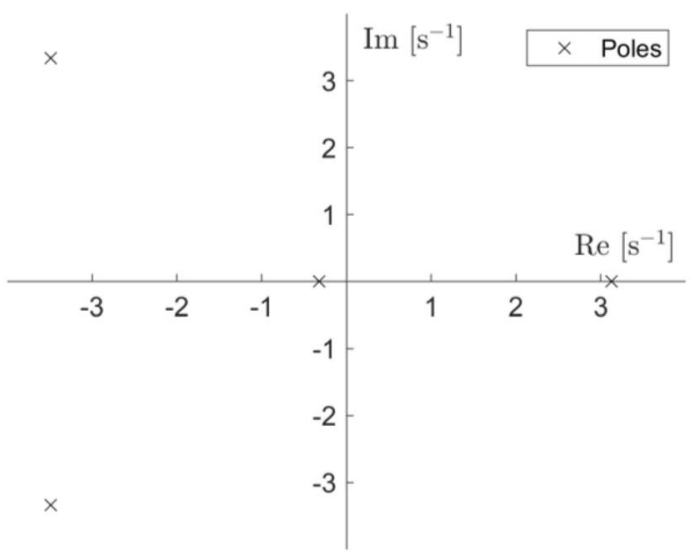

(a) Open-Loop System with the above-mentioned pitch moment and the rotation around the $y$-axis.

The vertical take-off is characterized by low vertical and horizontal velocities close to $w_{g}=u_{g}=0 \mathrm{~m} / \mathrm{s}$. In these states, the system requires a controller to be stabilized. By definition, the flight system of the Airborne Wind Energy has to endure high wind velocities. In hover mode at $w_{g}=0 \mathrm{~m} / \mathrm{s}$, the flying wing can reduce its pitch angle with the elevons to orientate its nose into the wind. Thereby, the component of the thrust vector in horizontal direction counteracts the drag of the wind velocity to maintain the vehicle's position. The striven goal is to show that the controller is able to stabilize the unstable poles during VTOL for the linearized model around the trim state at $w_{g}=u_{g}=0 \mathrm{~m} / \mathrm{s}$. However, a deeper analysis with limitations of the actuator, robustness and an overall performance of the controller for the whole velocity range is out of the range of this paper.

A simplified control approach is applied to stabilize the trim states of longitudinal motion during VTOL. The pitch angle $\Theta$ and its derivative the pitch rate $q$ of the flight system are fed back to modify the pitch moment by adjusting the elevon deflection $\kappa$, see Fig. 17.

A PD-controller feeds back the pitch angle $\Theta$ with factor $k_{\kappa \Theta}$ directly to a change in deflection angle $\kappa_{P}$ and the pitch rate $q$ with factor $k_{\kappa q}$ to a change in deflection angle $\kappa_{D}$. Both changes in deflection are summed up and serve as input for the flight system, see Eq. (4):

$\kappa=-K \cdot \vec{x}=-\left(\begin{array}{llll}k_{\kappa q} & k_{\kappa \Theta} & 0 & 0\end{array}\right)\left(\begin{array}{c}q \\ \theta \\ u \\ w\end{array}\right)$.

So far, a vertical take-off without transition is considered, and therefore, the commanded pitch rate $q_{C}$ equals zero. For

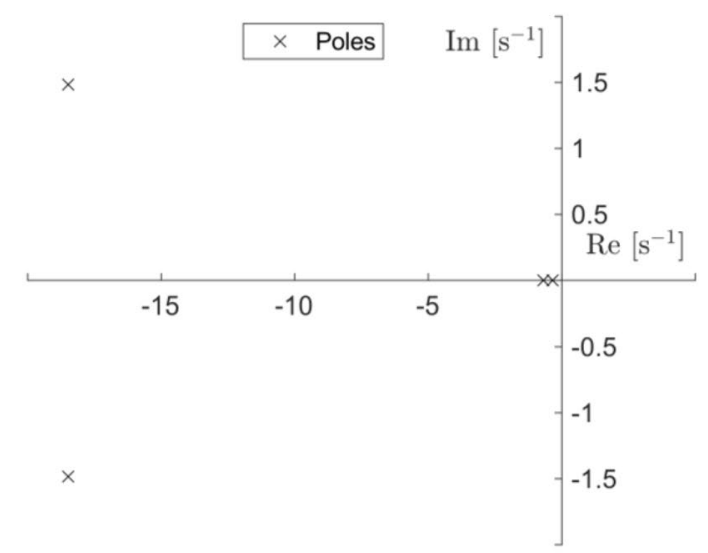

(b) Closed-Loop System

Fig. 16 Poles of the system at $w_{g}=u_{g}=0 \mathrm{~m} / \mathrm{s}$. a Open-loop system. b Closed-loop system 
Fig. 17 Closed-loop system of the longitudinal motion

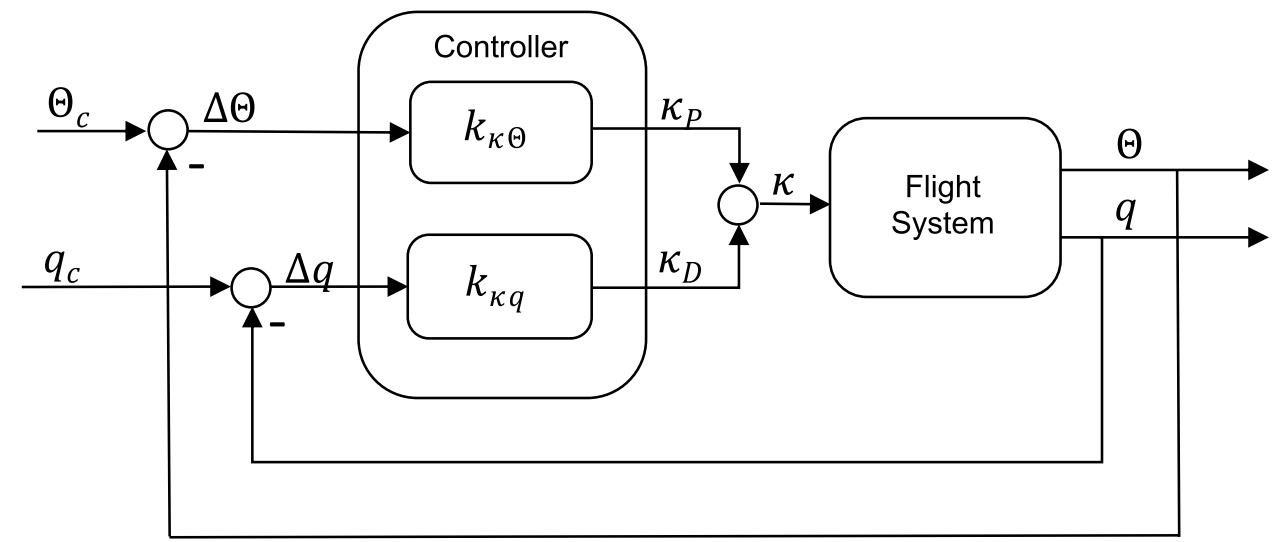

The velocity control is omitted a desired horizontal and vertical velocity, the corresponding pitch angle $\theta_{C}$ results from the condition of the trim state. The velocity control is omitted in Fig. 17, since the desired upward velocity is mapped directly to the thrust input.

For the linearized model, the closed-loop poles are analyzed with regard to their stability. Figure $16 \mathrm{~b}$ depicts the poles for the closed-loop system at $w_{g}=u_{g}=0 \mathrm{~m} / \mathrm{s}$. It consists of the linearized control model with $q$ and $\Theta$ fed back by the controller with factors $k_{\kappa \Theta}=5$ and $k_{\kappa q}=0.5$. All poles are in the left half plane and the controller is therefore able to stabilize the unstable poles during VTOL. Figure 18 depicts the time course of the vertical body-velocity $w_{b}$, the pitch rate $q$ and the corresponding elevon deflection $\kappa$ after an initial deviation of $w_{b}=1 \mathrm{~m} / \mathrm{s}$ from the hover state of $w_{g}=u_{g}=0 \mathrm{~m} / \mathrm{s}$. Due to the disturbance, a pitch moment is induced, resulting in a nose-down pitch rate $q$. Since the controller feeds back $q$ and $\Theta$ to the elevons, this deviation in $q$ results in a modification of the elevons deflection. A positive pitch moment is induced by the elevons deflection that decreases successfully pitch rate $q$ and body velocity $w_{b}$ back to zero, as it can be identified in the time-courses. The flight system can therefore reach its initial hover state with the control system.

\section{Conclusion and outlook}

This paper presents an analysis of the vertical take-off of a flying wing within the field of Airborne wind energy. The considered flying wing is able to control its motion around all three axes with its own elevons and its propulsion units. However, concerning the longitudinal motion during vertical take-off and especially hover flight, the flight envelope for states that can be trimmed is limited. Having a low inflow velocity, the effectiveness of the elevons is reduced as such that the pitch moments induced during maneuvers with intermediate horizontal but low vertical velocities cannot be trimmed by the untethered flying wing. However, these flight states are not part of the flight path of the untethered flight system. Nevertheless, this indicates that the flight system is not robust against high wind velocities during VTOL. This is not only decisive concerning wind robustness during take-off, but also the subsequent flight phase of the transition. In transition, a flight path from low to high horizontal velocities is aimed. The result of the flight envelope marks the area that can be flown through in quasi-stationary trimmed flight states. A larger trimmed

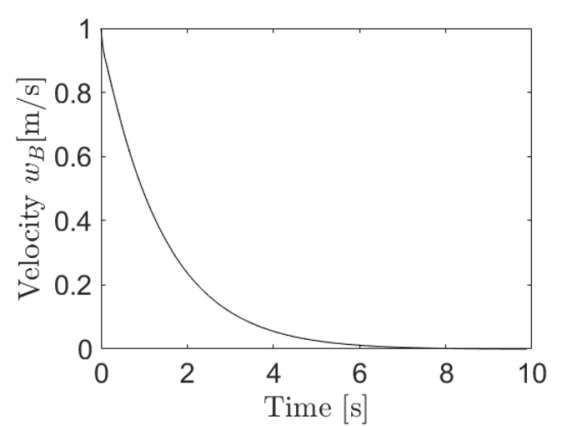

(a) Vertical Body-Velocity $w_{b}$

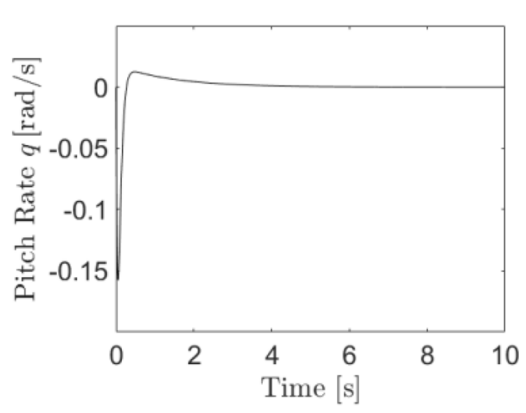

(b) Pitch Rate $q$

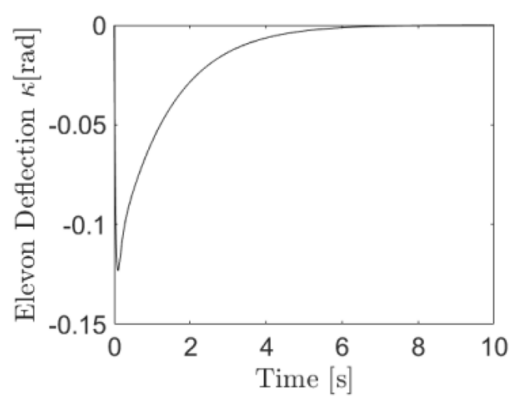

(c) Elevon Deflection

Fig. 18 Time-courses of the closed-loop system initialized at $w_{b}=1 \mathrm{~m} / \mathrm{s}$. a Vertical body-velocity $w_{b}$. b Pitch rate $q$. $\mathbf{c}$ Elevon deflection 
area at smaller upward velocities therefore allows a transition at lower upward velocities as well.

To increase this flight envelope, various modifications of the flying wing are investigated to identify their impact on the flight envelope for trimmed states. A higher elevon area and an adjustment of the thrust vector's point or angle of attack can enhance the flight envelope. These modifications increase the flight system's ability to induce a pitch moment and their impact is investigated. The strongest impact however, has the tether force. In the field of Airborne Wind Energy, the tether force can be utilized as further control variable to trim the flight system. The tether force is able to keep the flight system's position even at high horizontal wind velocities, leading to a smaller deviation of the flight system from the vertical reference attitude. This increases the flight envelope effectively. For strong tether forces however, the thrust limit of the flight system can be reached. Therefore, a shift of the tether force behind the center of gravity can induce the same equalizing pitch moment at lower tether forces. This allows a trimmed flight state for all horizontal and upward velocities up to the thrust limit. A further discussion of the trim states of the tethered VTOL as function of the wind velocity and the tether attachment point is given in [13].

Next to the flight system modifications, the analysis of the linearized model reveals that the trim states during vertical take-off are unstable. A simplified controller is designed to stabilize these states. As a PD-Controller, it feeds back the pitch angle and pitch rate to the elevon deflection. It is shown that an exemplary closed-loop system is stable during hover flight, neglecting the influence of actuator dynamics and omitting a detailed tuning of the controller.

This contribution focusses on the vertical take-off. To start the flight operations of energy generation, a trimmed flight path to transition from this vertical position into tethered crosswind flight has to be performed. This can also include a non-stationary transition, in which not all states are trimmed to a static equilibrium. At high wind velocities, there might be still an area of untrimmed states. Alternative flight paths like taking-off with span width in wind direction have to be investigated for these wind conditions. Still, a certain robustness for wind gusts from any direction is necessary, for which this contribution offers a detailed analysis of the possible flight range of velocities and possibilities for enlargement.

Concerning the constructive modifications of the tether force, the influence during flight is still to be investigated. Besides, the results depend on the model of the flight system and have to be verified in flight tests. Assumptions were made regarding the inflow velocity of propeller, elevon and aerodynamic model. To calculate the slipstream of the propeller, only the axial velocity is considered and aerodynamic polars are extrapolated to $180^{\circ}$. As further turbulence and flow separation are expected at high angles of attack, wind tunnel experiments and flight tests will be conducted to validate the data. Next to the longitudinal motion, the lateral motion is to be respected as well and the controller extended to control the position of the flight system taking-off from the ground until the initiation of the energy operation phase.

Acknowledgements We would like to thank all project partners for their work and contributions to the EnerGlider project. Special thanks are due to the Federal Ministry of Economic Affairs and Energy (BMWi) for the funding and to the Project Management Jülich for the support and coordination of the EnerGlider project.

Funding Open Access funding enabled and organized by Projekt DEAL. The project is funded by the Federal Ministry of Economic Affairs and Energy of Germany (BMWi).

Availability of data and material Raw data were generated at RWTH Aachen University. Derived data supporting the findings of this study are available from the corresponding author on request.

Code availability Code was generated at RWTH Aachen University and is subject to their intellectual property.

\section{Declarations}

Conflict of interest No potential competing interest.

Open Access This article is licensed under a Creative Commons Attribution 4.0 International License, which permits use, sharing, adaptation, distribution and reproduction in any medium or format, as long as you give appropriate credit to the original author(s) and the source, provide a link to the Creative Commons licence, and indicate if changes were made. The images or other third party material in this article are included in the article's Creative Commons licence, unless indicated otherwise in a credit line to the material. If material is not included in the article's Creative Commons licence and your intended use is not permitted by statutory regulation or exceeds the permitted use, you will need to obtain permission directly from the copyright holder. To view a copy of this licence, visit http://creativecommons.org/licenses/by/4.0/.

\section{References}

1. Ampyx Power: Landing deck with rotation system being readied for manufacturing. https://www.ampyxpower.com/news/landingdeck-with-rotation-system-ready-for-manufacturing/. Accessed Jul 2020

2. Enerkite Technologie: https://www.enerkite.de/technologie.html. Accessed Aug 2020

3. KiteGen: Taking-Off, Flying and Landing in Safety (2012). http:// www.kitegen.com/en/2012/03/13/taking-off-flying-and-landi ng-in-safety/

4. Ferrell, P., Smith, B., Stark, B., Chen, Y.: Dynamic flight modeling of a multi-mode flying wing quadrotor aircraft. In: International Conference on Unmanned Aircraft Systems (ICUAS), pp. 398-404, IEEE (2013)

5. Verling, S., Weibel, B., Boosfeld, M., Alexis, K., Burri, M., Siegwart, R.: Full attitude control of a VTOL tailsitter UAV, IEEE 
international conference on robotics and automation (ICRA), pp. 3006-3012, IEEE (2016)

6. Liang, J., Fei, Q., Wang, B., Geng, Q.: Tailsitter VTOL flying wing aircraft attitude control. In: 31st Youth Academic Annual Conference of Chinese Association of Automation (YAC), pp. 439-443, IEEE (2016)

7. Hartmann, P.: Predictive flight path control for tilt-wing aircraft, Ph.D. Dissertation, Faculty of Mechanical Engineering at RWTH Aachen University (2017)

8. Duda, D. F., Fuest, H., Islam, T., Moormann, D.: Hybrid modelling approach for the tether of an airborne wind energy plant, Deutscher Luft- und Raumfahrtkongress (2020)

9. Schütt, M., Islam, T., Hartmann, P., Moormann, D.: Scalable design approach to analyze flight mechanical performance for tiltwing UAVs, International Council of Aeronautical Sciences, ICAS (2018)

10. Montgomerie, B.: Methods for root effects, tip effects and extending the angle of attack range to $+/-180$, with application to aerodynamics for blades on wind turbines and propellers. Swedish Defence Research Agency (2004)

11. Binz, F., Moormann, D.: Actuator Modeling for Attitude Control Using Incremental Nonlinear Dynamic Inversion. In: International Micro Air Vehicle Conference, Madrid (2019)

12. McCormick, B.W.: Aerodynamics, aeronautics, and flight mechanics. Wiley, New York (1994)

13. Fuest, H., Duda, D. F., Islam, T., Moormann, D.: Flight control architecture of a flying wing for vertical take-off and landing of an airborne wind energy system. AIAA Scitech 2021 Forum, pp. 1816, Nashville (2021)

Publisher's Note Springer Nature remains neutral with regard to jurisdictional claims in published maps and institutional affiliations. 